\title{
Favorable outcome of COVID-19 infection in a patient with congenital myasthenic syndrome
}

\author{
Silvia Bonanno ${ }^{1}$ (i) $\cdot$ Lorenzo Maggi ${ }^{1}$ (D) \\ Received: 30 September 2020 / Accepted: 12 January 2021 / Published online: 22 January 2021 \\ (C) Fondazione Società Italiana di Neurologia 2021
}

Keywords COVID-19 $\cdot$ Congenital myasthenic syndrome $\cdot$ Neuromuscular disorders $\cdot$ Positive outcome

\section{Background}

Patients affected by neuromuscular disorders (NMDs) are theoretically at higher risk for severe illness from SARS-CoV2 due to respiratory and swallowing muscle weakness [1]. Here we describe the first case of congenital myasthenic syndrome (CMS) who was infected with COVID-19 and showed a positive outcome.

\section{Case}

A North-African female presented with eyelid ptosis and ophthalmoparesis since the age of 4 months, followed by generalized fatigable weakness with significant impairment in common daily activities. At the age of 13 , she was diagnosed with a congenital myasthenic syndrome caused by a homozygous mutation on the gene encoding the AChR epsilon subunit (CHRNE; c.1121-1127dup; p.E376DfsX2). Over the years, she was treated with pyridostigmine $60 \mathrm{mg}$ 4 times daily, salbutamol $4 \mathrm{mg} 3$ times daily, and 3,4diaminopyridine $(3,4-\mathrm{DAP})$ at the maximum dose of $80 \mathrm{mg}$ daily in four administrations. Patient's medical history included obesity and allergic-asthma, treated with tiotropium bromide and formoterol fumarate inhalation powders. At February 2020, at the age of 21, patient's myasthenia gravis activity daily living score (MG-ADL) [2] was $13 / 24$ (speech $=1$, chewing $=1$, swallowing $=2$, breathing $=1$, ability to wash hair/brush teeth $=2$, ability

Lorenzo Maggi

lorenzo.maggi@istituto-besta.it

1 Neuroimmunology and Neuromuscular Diseases Unit, Fondazione IRCCS Istituto Neurologico "Carlo Besta", Via Celoria 11, 20133 Milan, Italy to stand up from a chair $=2$, diplopia $=2$, eyelid ptosis $=$ 3). Last March, she experienced fever over $38^{\circ} \mathrm{C}\left(100^{\circ} \mathrm{F}\right)$ accompanied by severe tiredness and taste loss. Residing in one of the COVID-19 outbreak epicenters in north of Italy, the patient self-quarantined at home. She had no clear exposure history to sick subjects. At that point, she was not tested for the presence of SARS-CoV2 genome, and neither she underwent blood tests and chest $\mathrm{x}$-ray. She took oral paracetamol and clarithromycin following her primary physician indications, along with her ongoing therapy for the treatment of CMS (pyridostigmine $60 \mathrm{mg} 4$ times daily, salbutamol $4 \mathrm{mg} 3$ times daily, and 3,4-DAP $20 \mathrm{mg} 4$ times daily) and asthma (tiotropium bromide and formoterol fumarate, 2 puff each, 3 times daily). MG-ADL score performed through telephone consultation was $14 / 24$, with a 1 point worsening in the ability to wash hair/brush teeth $(=3)$. Patient recovered from fever in 3 days, and loss of taste resolved in 20 days, without complaining any relevant worsening of CMS symptoms, except for the aforementioned severe tiredness. Few weeks later, her brother presented with pneumonia and fever up to $40{ }^{\circ} \mathrm{C}\left(104^{\circ} \mathrm{F}\right)$ with a positive screening for COVID-19 genome positive. Afterwards, the patient underwent a serological test for anti-SARS-CoV antibodies, and $\mathrm{IgG}$ index resulted 5.64 (S/C), where the threshold for positivity was $>1.40$; COVID-19 RTPCR test was negative. No test for IgM antibodies was performed.

The patient was newly evaluated at the neurology outpatient clinic at the beginning of August; she reported an almost complete recovery from COVID-19 infection, except for a lasting mild tiredness. Blood test, respiratory function tests, and neurologic examination did not detect any relevant complication, beyond the underlying CMS. MG-ADL score was 13/24, comparable to February 2020. Furthermore, MG Composite (23/50) [3] and the Fatigue Severity Scale (55/ 63) [4] did not change compared to February 2020. 


\section{Discussion}

As other NMDs, CMS are at high risk of complications in case of COVID-19 [1]. In addition, CMS are well-known to be acutely worsened by trigger factors, mainly fever and infections, also causing life-threatening events [5]. Here, we present the first CMS case who has been infected with SARS-CoV2 and recovered with no complications. Despite the patient's baseline bulbar mild weakness and asthma, she did not develop severe infection with acute respiratory syndrome, and she did not experience a relevant deterioration in ocular, bulbar, and limb muscle weakness. Considering the MG-ADL minimal worsening during the infectious event, the patient's tiredness appears to be more related to the COVID-19 infection itself, rather than CMS worsening. Besides, CHRNE patients are not the most prone to worsening provoked by infections and fever compared to other CMS as those related to mutations in RAPSN and CHAT genes [5, 6]; this may partially explain the low infection-impact on patient's clinical status. In this view, fever and infections may also trigger worsening in patients affected by myasthenia gravis, although data recently reported in literature did not support a worse outcome even in these patients [7].

\section{Compliance with ethical standards}

Conflict of interest S.B. and L.M. have no financial disclosure and declare no conflict of interests.
Ethical approval The Authors have read the Journal's position on issues involved in ethical publication and affirm that this report is consistent with those guidelines.

\section{References}

1. Guidon AC, Amato AA (2020) COVID-19 and neuromuscular disorders. Neurology 94(22):959-969. https://doi.org/10.1212/WNL. 0000000000009566

2. Wolfe GI, Herbelin L, Nations SP, Foster B, Bryan WW, Barohn RJ (1999) Myasthenia gravis activities of daily living profile. Neurology 52(7):1487-1489. https://doi.org/10.1212/wnl.52.7.1487

3. Burns TM, Conaway M, Sanders DB, MG Composite and MGQOL15 Study Group (2010) The MG composite: a valid and reliable outcome measure for myasthenia gravis. Neurology 74(18):1434 1440. https://doi.org/10.1212/WNL.0b013e3181dc1b1e

4. Alekseeva TM, Gavrilov YV, Kreis OA, Valko PO, Weber KP, Valko Y (2018) Fatigue in patients with myasthenia gravis. J Neurol 265(10):2312-2321. https://doi.org/10.1007/s00415-0188995-4

5. Maggi L, Bernasconi P, D'Amico A et al (2019) Italian recommendations for diagnosis and management of congenital myasthenic syndromes. Neurol Sci 40(3):457-468. https://doi.org/10.1007/ s10072-018-3682-x

6. Finsterer J (2019) Congenital myasthenic syndromes. Orphanet journal of rare diseases 14(1):57. https://doi.org/10.1186/s13023-019$1025-5$

7. Ramaswamy SB, Govindarajan R (2020) COVID-19 in refractory myasthenia gravis- a case report of successful outcome. J Neuromuscul Dis 7(3):361-364. https://doi.org/10.3233/JND200520

Publisher's note Springer Nature remains neutral with regard to jurisdictional claims in published maps and institutional affiliations. 